\title{
The Production of Single Crystals of Metals and some of their Properties. ${ }^{1}$
}

\author{
By Prof. H. C. H. Carpenter, F.R.S.
}

\section{$\mathrm{T}$}

HE problem of preparing a piece of metal in the form of a single crystal may be approached in one of two ways. As ordinarily prepared, metals and alloys consist of a large number of small crystals the size of which varies in the great majority of cases from about 100,000 to several million in' a cubic inch. These result from the fact that when the metal or alloy freezes, crystallisation takes place at a large number of centres and the crystals grow until they meet one another. The boundaries, therefore, are produced by the meeting of these crystals and vary very much in shape. The net result is rather similar to that of a jig-saw puzzle, for the crystals are oriented quite at haphazard. The shapes and sizes of these crystals may be altered by working and heat-treatment, and the precise rearrangement may go so far as to result in the birth of new crystals, but the net result is always an aggregate of comparatively small crystals.

The properties of metals and alloys are the properties of these small crystals, and are due to two factors- $(a)$ the crystal itself, and $(b)$ the crystal boundary. In the crystal itself the atoms are arranged in a particular pattern corresponding to the symmetry of the metal. At the boundary, on the other hand, this condition does not obtain. For example, where two crystals are in contact atoms meet in two different directions, while at the place where three crystals touch, atoms meet in three different directions, and so on. It has been known for some time that the crystal boundary is stronger than the metal crystal, in the sense that normally when a metal is fractured it breaks through the crystal and not along the boundary, and the fracture of a metal test-piece is the summation of a large number of fractures running through small crystals. Hitherto it has been impossible, except in a very few isolated cases, to determine the properties of a metal crystal apart from those of the crystal boundary. The great and indeed fundamental interest of the problem of preparing a piece of metal in the form of a single crystal, therefore, is that its solution would enable the properties of the metal crystal to be determined.

\section{Single Crystals Formed DiRect From the Liquid.}

Evidence has been in existence for some time that, provided that the right conditions obtain, very large crystals can be grown from the liquid. The famous Tschernoff crystal dates back to about I880. It was a large iron crystal, which he noticed in the 'riser' (head) of an ingot at the Vickers' works in Sheffield, and was upwards of 6 in. in length. Its shape was peculiar, and was what is called a 'crystal skeleton'i.e. it had grown along the crystallographic axes to a greater extent than the filling-in process had occurred, and the net result was something which looked very like the branch of a pine-tree. Other instances of the accidental occurrence of large crystals have been

\footnotetext{
2 From the sixteenth May Lecture to the Institute of Metals, delivered on May r.
}

NO. 2964 , VOL. I I 8] noticed by Osmond and Cartaud, Moellendorff and Czochralski.

So far as I have been able to discover, the problem of preparing a single crystal direct from the liquid was first solved by Czochralski in I9I8. He prepared long thin threads of crystals by drawing out a rod at a particular rate from the molten metal. As the rod was withdrawn the metal solidified and the resulting wire $(0.5 \mathrm{~mm}$. diameter) was found to consist of one crystal. Success was thus achieved with tin, lead, and antimony. Later Gomperz improved the technique of Czochralski's method. He used a silica rod on which to deposit the crystal growing from the melt and surrounded this with a neutral gas.

Quite recently (1923 and $\mathrm{r}_{925}$ ) P. W. Bridgman has prepared much larger single crystals by melting the metal in a tube tapered at one end to a point and lowered through the furnace at a given rate. He found that if the lowering is at a speed less than the velocity of crystallisation and slow enough for the heat of solidification to be dissipated by conduction, then the metal will usually crystallise as one grain, provided that only one nucleus started at the bottom of the tube. In this way he prepared crystals of antimony, bismuth, cadmium, tellurium, tin, and zinc. It is particularly interesting to notice that this method also holds for metals having a polymorphic transition point (tin and antimony).

The melting was carried out in vacuo, and great stress is laid on the importance of freedom from dirt as an essential condition of success. At some little distance from the bottom the tube was constricted to a capillary, O. I mm. in diameter. It was found that one orientation of the crystals is usually more favourable for growth than others, so that even if crystallisation starts from more than one centre at the bottom, that crystal will eventually win through the capillary which is most favourably situated. Bridgman found that the most favourable orientation is, in almost all cases, when the plane of easiest cleavage or slip is parallel to the axis of the casting. In this way single crystal rods up to a diameter of $2 \cdot 2 \mathrm{~cm}$. were prepared. The same device was adopted by Obreimow and Schubnikow.

Valuable work on the single crystal wires produced by Czochralski's method and their distortion by mechanical stress has been carried out by Polanyi, Schmid, and Schouborn, while Bridgman has determined a number of physical properties of his single crystals of metals, which were purposely chosen because they did not possess the highest symmetry and therefore might be expected to give different values in different directions. This expectation was fulfilled.

\section{Single Crystal Test-Pieces from Pre-Existing Crystals.}

Here, again, a study of the literature showed it to contain a number of observations which indicated that, provided that the right conditions were obtained, success might be achieved in this direction, although the chances did not appear so good as that of preparing 
a single crystal from the melt. Sauveur was, I think, the first investigator to show that, by carefully straining and afterwards heating metals, crystals of a much larger size than the normal could be produced, and stated that there was a critical stress which produced the largest crystals. Afterwards Ruder, Chappell, Jeffries, and Hanson showed that if a metal is locally deformed and then heated, exceptionally large crystals form at some distance from the point at which stress is the severest. If a tapered test-piece was used a 'strain gradient' was obtained, and it was found that the largest crystals always formed within the strained region, but farther from the area of greatest strain the higher the temperature. Seligman and Williams, working in my laboratory, stretched aluminium sheet, which had been previously heated, to various degrees, and found on heating that, up to a certain point, a small deformation had no effect. Beyond this, however, large crystals were formed, and as the deformation was further increased the crystal size was diminished. The crystals produced by these methods were very large compared with those in the original metal, some of them being 0.5 in. long.

In recent years wires have been produced, originally by accident, both of tungsten and molybdenum, in which crystals occupy the entire cross-section of the wire. This is done by drawing the metal through dies at a certain rate and temperature. Sykes, experimenting on molybdenum wire, obtained crystals 0.5 in. long, with an average diameter of 0.013 in.

Experiments on the production of single aluminium crystals were begun several years ago by Miss C. F. Elam, my research assistant, and myself. For many months we studied the structural changes produced in a crystal aggregate by deformation followed by heat, which may be summarised as follows : The first effects of strain are revealed by the presence of slip-bands, and in some cases of twin crystals. The former are completely, and the latter to some extent, removed by heating. No change is observed in the shape of the crystals. Somewhat greater deformation, however, followed by heat, produced actual crystal growth, and at this stage the boundaries of the crystals became active. This activity is shown in the capacity of the growing crystal to push forward its boundaries in certain directions, thus invading other crystals, upon which it imposes its orientation. A still greater deformation produces a change which appears to take place exclusively in the boundaries of the deformed crystals. It is here that new crystals are born, possessing a different orientation and a much smaller size. Accordingly, in this third stage there is a refining of the crystal structure. From the point of view, therefore, of producing large crystals from an aggregate of small ones, the intermediate degree of strain, just referred to, is the important one.

The increase in crystal size could be clearly followed in our experiments with an alloy of tin containing a little antimony, where the actual movement of the crystal boundary was observed and photographed. Our experiments further showed that in the absence of a change of phase neither crystal growth nor recrystallisation will take place in a metal unless it has been plastically deformed and afterwards heated to a certain minimum temperature for a certain minimum time.
They also showed that there is no gradual increase in size from the original crystals up to the largest, but that the latter appear to form directly from them. It is clear therefore that, in the production of large metal crystals, the adjustment between mechanical strain and the temperature of heating is extremely important.

This point can be clearly illustrated in the case of the metal aluminium. Test-pieces, after preliminary heattreatment to remove work hardness and render the crystals so far as possible equiaxed, were subjected to varying degrees of strain. After this they were all heated to $55^{\circ} \mathrm{C}$. for 6 hours. Finally, they were etched in a ro per cent. solution of caustic soda. Below the minimum strain required for growth, the crystal size remains unaltered. At the critical strain large crystals suddenly appear. Above this the size diminishes in proportion as the strain increases.

\section{Production of Single Crystals in Sheets.}

By this time we were ready to approach the problem itself, and our first experiments aimed at converting the crystals in the parallel portion of a test-piece 4.0 in. $\times I \cdot 0$ in. $\times 0.125$ in. of aluminium into a single crystal. Calculation showed that the total number of crystals contained in the parallel portion of the test-piece was about $\mathrm{I}, 687,000$.

After many months of work success was achieved in the following way: The test-piece must, in the first place, be accurately machined along the parallel portion. In order to convert this into a single crystal three treatments are necessary : the first thermal, the second mechanical, the third thermal. The first treatment is necessary to soften the metal completely and produce new equiaxed crystals of so far as possible uniform size, the average diameter being $\frac{1}{150}$ in. The second consists in straining these crystals to the required amount, and the third in heating the strained crystals to the requisite temperature, so that the potentiality of growth conferred by strain could be brought fully into operation.

The most suitable temperature of the first heating was found to be $55^{\circ} \mathrm{C}$., and the time 6 hours. The precise degree of strain for the aluminium used was an elongation of $x \cdot 6$ per cent. on 3 in. produced by a stress of 2.4 tons per sq. in. The final heat-treatment was begun at $450^{\circ} \mathrm{C}$., and the temperature raised at about $25^{\circ} \mathrm{C}$. per day up to $550^{\circ} \mathrm{C}$. It was then brought finally up to $600^{\circ} \mathrm{C}$. for one hour, in order to complete the absorption of small crystals on the surface, which persistently remained at lower temperatures. On an average, one test-piece in four is converted into a single crystal in this way. The boundary at each end extends in the form of an irregular line into the wide head of the testpiece. The time required for the single crystal to grow in this way is therefore about roo hours.

The conditions laid down by us for the production of a single crystal were that every crystal in the complex should be strained a certain small amount, and that one of them should be strained rather more than all the rest. We were originally disposed to think that the crystal which grew and absorbed all the others was the one most highly energised by strain. Later investigations with X-ray analysis, however, have shown that

$$
\text { NO. } 2964 \text {, VOL. I I } 8]
$$


single crystals produced in this way are free from strain, and we have accordingly modified our view and consider that the most highly strained crystal deposits a nucleus free from strain upon which all the other crystals ultimately align themselves, thus producing an unstrained single crystal.

\section{Production of Single Crystals in Bars.}

We next proceeded to apply these methods to the production of single crystals in round bars. We found that, provided that the same conditions as to accuracy of machining, correct crystal size, degree of straining, and final heat-treatment were maintained, single crystals could also be produced in round-bar test-pieces, both in diameters of 0.564 in. and 0.798 in. Single crystal test-pieces 9 in. long and 0.564 in. in diameter have been prepared in this way.

Deformation of these test-pieces under tensile stress is very remarkable. On one hand, the 'normal' bar, consisting of small crystals, drew down with a roughening of the surface, a cup and cone fracture, and the maintenance of a circular cross-section throughout. On the other hand, when a single crystal was pulled in this way it produced an ellipse. As the test proceeded the ellipse became narrower and narrower in one diameter, while the other diameter remained nearly constant. Presently a point was reached when a characteristic lens-shaped figure was formed. As the bar pulled apart this became smaller and smaller. Eventually fracture took place at each side and ultimately in the middle.

The final result was that the fractured surface of each half of the test-piece was a very acute ellipse or wedge, the metal being grooved in each case. Characteristic flow lines also made their appearance on the testpieces, corresponding to the slipping of the metal in this remarkable and quite novel way. They may be called ' glide-ellipses.' Here, again, the properties varied considerably in different specimens, the tenacities ranging from $3^{\circ} \circ$ to $4^{\circ} \circ$ tons per sq. in. and the elongation from 57 to 85 per cent. In some of these experiments the volume of the single crystal exceeded 2 cu. in., which means that about $7,000,000$ crystals had coalesced in their formation.

It has not been found possible to produce single crystal test-pieces of square bars. Owing to the shape, these fail to strain uniformly, and, on heating, crystals grow from the four corners and meet in a line in the middle of the four sides. Some of the crystals were from 2 in. to 3 in. long and were visible on two adjacent sides. Remarkable effects were obtained on breaking them. In some places the corners became rounded, while in others they were narrowed to a ridge of almost knife-edge thickness. The whole bar became very much twisted and distorted.

\section{Analysis of the Distortion of Single Crystal Bars.}

The distortions of the single crystal test-pieces produced both in sheet and bar forms were very remarkable, and clearly suggested that the crystal axes were not oriented in the same direction in each specimen. In discussing this matter with $\mathrm{Mr}$. (now Prof.) G. I.
Taylor, he thought that it would be a straightforward, though possibly laborious, task to determine the relationship between the orientation of the axes and the distortion produced in a tensile test, and that by examining a number of specimens some general results might be obtained about the forces necessary to produce distortions of this type. He kindly agreed to join us in the work of testing these matters. Before the test contemplated could be carried out, however, it was necessary to obtain the help of an expert in crystal analysis by X-rays. Dr. A Müller accepted our invitation, and succeeded in devising a satisfactory method of determining the orientation of the crystal axes in single crystal bars by means of X-rays.

For the purpose in hand it was necessary to work with a square bar. As already mentioned, single crystals cannot be grown in square bar form. We had, therefore, to produce a round single crystal test-bar and then to machine it down until its section was square. The method of investigation adopted was as follows : Each face was marked by a scratch parallel to the length of axis of the specimen and by cross scratches at 0.5 in. intervals. The faces were numbered I, 2,3 , and 4 , so that when the specimen was placed upright in the testing machine the faces appeared in this order when the observer moved round the machine in an anti-clockwise direction. At each successive stage of the test the extension between each pair of cross marks was measured on each face. At the same time the angles between the cross scratches and the longitudinal scratch were measured in each case. In addition, the thickness of the specimens between pairs of opposite faces and the angle between neighbouring faces were also measured. These suffice to determine the nature of the distortion.

Measurements showed that near the ends of the specimen, where it was held in the grips of the testing machine, the measurements were not quite the same as those near the middle, but that the central portion was nearly uniformly strained. There is a high degree of uniformity among the measurements of extension in the three middle compartments of each face, especially in the earlier stages of the test.

Measurements were made at extensions of 5, ro, 15 , $20,3 \circ, 40,60$, and 78 per cent., when the crystal broke. As the test proceeded the square section of the bar gradually disappeared, and the final result was $a$ parallelepiped of remarkable form. The cross scratches, although remaining rectilinear, shifted by a definite angle which became markedly acute. When the specimen finally broke, it contained two very acute and two very obtuse angles.

Prof. Taylor and Miss Elam followed up this work with a paper published in 1925. In this they have described similar experiments made with several more specimens in order to find out whether the conclusions previously reached are general in their application and to settle several points which the previous experiments left in doubt. The chief result of their earlier work was to show that of the twelve crystallographically similar possible modes of shearing, the one for which the component of sheer-stress in the direction of shear was greatest was the one which actually occurred. The first object of the later work was to determine the orientation of the crystal axes of several specimens.

NO. 2964, VOL. I I 8 ] 
Assuming that the relationship outlined above holds in general, it is possible to predict the orientation of the plane on which slipping should occur. Three more single crystal test-pieces were stretched and their distortions analysed by the method already described. It was found that in each case the distortion was such as would be produced by shearing parallel to a single plane, and that this plane of slip was the one predicted. It was also found that the changes in orientation of the crystal axes relative to the axis of the specimen during the test were in good agreement with the prediction.

In the previous work it was not possible to make a detailed analysis of the deformation during the last stages of the stretching, but it was shown that the deformation ceased to be due to slipping on one crystal plane. Reasons were given for supposing that the deformation might be due to slipping on two crystal planes simultaneously, and it was shown that the crystal axis which formed the intersection of these two planes remained unstretched during the test, as it should if the distortion were in fact due to this cause. There are, however, a number of other types of distortion which might also leave this particular axis unstretched. In their later paper Taylor and Elam showed that for a small distortion the 'unstretched' cone passes through or very close to three other crystal axes, which, in fact, determine all the possible kinds of unstretched cone which can be produced by double slipping of the type contemplated. This completes the proof that the slipping in the last stages of the test is of this type.

When the crystal begins to slip on a second plane it seems likely that the rate of slip on each plane would be the same. In this way they would remain inclined at equal angles to the axis of the specimen, but it was impossible to verify this suggestion from the previous experiments. The second paper contains the test of this, and it is found that, although double slip does begin when two planes get to the position in which they make equal angles with the axes, the rate of slipping on the original slip plane is sometimes greater than it is on the new one. The two planes do not, therefore, necessarily remain at equal angles with the axis, but the process cannot be followed very far because the specimen usually breaks when only a comparatively small amount of double slipping has occurred.

A most interesting test described in this paper was that in which the authors distorted a single crystal test-piece, in which they predicted that from the original position of the crystal axes double slipping should begin at once. A specimen was found the axes of which corresponded very closely to this position, and it was found that double slipping did occur during nearly the whole extension. In this case it was found that the amount of slipping on the two planes was practically equal, and that the axis of the specimen remained very close to the (II2) axis during the whole test.

A point of which Taylor and Elam made special mention was the extraordinary uniformity of the stretched specimens. They showed a photograph of specimen No. 68 which had been stretched to an extension of 70 per cent. To the eye it appears absolutely uniform. They gave numerical data as to the varia- tions in extension of different parts of the specimen, and found that the average variation of $\epsilon$ was 0.0028 during the first 5 per cent. Up to 20 per cent. extension the variation did not exceed this figure; it then began to increase, until at the breaking point it rose to 4 parts in 100.

The chief conclusions drawn by the authors from the theory of fracture contained in their paper are-(r) that with a stress-strain curve of the type found by them, fracture of aluminium single crystals cannot occur by slipping on a single crystal plane, and (2) that the geometrical conditions alone imply that fracture takes place more easily when double slipping occurs than when all the slipping is confined to one plane.

Miss Elam and I have recently investigated the distortion of single crystal test-pieces of aluminium followed by subsequent heating. We have found that the crystals can be deformed so much as 7 per cent. without being destroyed on heating. Beyond this amount the metal recrystallises and breaks up into a number of smaller crystals the size of which depends on the amount of distortion. Near the fracture the crystals are smallest and they increase in size towards the shoulders of the test-piece. The recrystallised metal shows exceptionally straight crystal boundaries, and the crystals are frequently twinned.

We then made a detailed examination of the behaviour of two round single crystal test-pieces after successive increments of strain followed by heating. The orientation of the bar was first determined by means of X-rays, and the metal then extended 5 per cent. The position of the crystal axes was then determined, and it was found that the reflections on the photographic plate were not so well defined and were spread over a large reflecting angle. The crystal was then heated to $400^{\circ}, 500^{\circ}$, and $600^{\circ} \mathrm{C}$. and examined after various periods at these temperatures. No change appeared after this treatment. The nature of the X-ray reflections was unaltered, and there was no change in the position of the reflecting planes. After a second extension of 5 per cent. the positions of the reflecting planes were redetermined and the bar heated to $350^{\circ}$ and $450^{\circ} \mathrm{C}$. No change was observed. After heating at $550^{\circ} \mathrm{C}$. the reflections that had been found previously had disappeared, but new ones were obtained in other positions.

A comparison of the reflecting planes from different parts of the bar, including opposite sides of the same reference plane, showed that it was still a single crystal, but that it possessed an entirely different orientation from that of the original. Moreover, the X-ray reflections now obtained were sharp and well defined as in the original crystal. Etching in caustic soda revealed a homogeneous structure. Further extension of 5 per cent. produced results similar to the first extension, and heating up to $600^{\circ} \mathrm{C}$. produced no change. On heating after the fourth extension, however, the orientation was found to have changed once more, but the bar still remained a single crystal. The process was again repeated, and after the sixth extension a partial recrystallisation took place. Another single crystal test-piece was subjected to this treatment with exactly similar results. Here again the crystal moved successively into two new positions. 